\title{
Association of PPARG Pro12Ala polymorphism with insulin sensitivity and body mass index in patients with polycystic ovary syndrome
}

\author{
DINKA PAVICIC BALDANI ${ }^{1}$, LANA SKRGATIC ${ }^{1}$, JASMINA Z. CERNE $^{2}$, \\ POLONCA FERK $^{3}$, VELIMIR SIMUNIC ${ }^{1}$ and KSENIJA GERSAK ${ }^{2,4}$
}

\begin{abstract}
${ }^{1}$ Department of Obstetrics and Gynaecology, Division of Human Reproduction, University of Zagreb Medical School, University of Zagreb Medical Centre, Zagreb, Croatia; ${ }^{2}$ Department of Obstetrics and Gynaecology, Division of Medical Genetics, University of Ljubljana Medical Centre, Ljubljana; ${ }^{3}$ Department of Pharmacology and Experimental Toxicology, Faculty of Medicine, University of Maribor, Maribor; ${ }^{4}$ Department of Obstetrics and Gynaecology, Faculty of Medicine, University of Ljubljana, Ljubljana, Slovenia
\end{abstract}

Received October 21, 2013; Accepted November 13, 2013

DOI: $10.3892 /$ br.2013.215

\begin{abstract}
Insulin resistance is one of the key factors in the pathogenesis of polycystic ovary syndrome (PCOS). The peroxisome proliferator-activated receptor gamma (PPARG) plays a role in the regulation of insulin sensitivity. The aim of the present study was to establish a possible association of the PPARG Pro12Ala polymorphism with PCOS and its effect on family and personal history, as well as on the metabolic and endocrine parameters in PCOS patients. A total of 151 PCOS patients and 179 healthy women of reproductive age were enrolled. History, body mass index (BMI), waist-to-hip ratio and the presence of phenotypic hyperandrogenism were recorded. Hormonal, metabolic and biochemical profiles were assessed. A molecular analysis for the genetic polymorphism was performed. One third (29.8\%) of the PCOS patients were found to be carriers of at least one variant of the Ala allele (X/Ala), while $70.2 \%$ carried two wild-type Pro alleles (Pro/Pro), with an equal distribution observed in the control group. The PCOS patients carrying the X/Ala alleles exhibited lower serum fasting insulin levels, homeostatic model assessment of insulin resistance (HOMA-IR) and BMI compared to Pro/Pro carriers. This finding was significant only in the lean PCOS group. The polymorphic genotype exerted no effect on history, hormonal and clinical hyperandrogenism, lipid status or C-reactive protein, leptin, adiponectin, resistin and ghrelin
\end{abstract}

Correspondence to: Professor Ksenija Gersak, Department of Obstetrics and Gynaecology, University of Ljubljana Medical Centre, 3 Slajmerjeva Road, Ljubljana SI-1000, Slovenia

E-mail: ksenija.gersak@mf.uni-lj.si

Key words: peroxisome proliferator-activated receptor gamma, polymorphism, polycystic ovary syndrome, glucose metabolism, reproductive hormones serum levels in women with PCOS. In conclusion, although the PPARG Pro12Ala polymorphism is not a major determinant of PCOS in the Croatian population, it may exert a positive effect on insulin sensitivity and BMI. As these associations were recorded exclusively in the lean group of patients with PCOS, this polymorphism potentially contributes to a protective role against hyperinsulinemia and obesity.

\section{Introduction}

Polycystic ovary syndrome (PCOS) is the most common endocrine disorder in women of reproductive age. It is characterized by exaggerated production of androgens and displays gross abnormalities in ovarian function and morphology (1). Various PCOS phenotypes presumably result from the interaction between multiple predisposing genomic variants, each exerting only minor effects and strong environmental influences (2). As PCOS is associated with obesity, insulin resistance and compensatory hyperinsulinemia (3), disturbed secretion of adipokines (4) and an elevated risk for diabetes mellitus type 2 (T2DM) (5) and cardiovascular diseases (CVD) (6), the peroxisome proliferator-activated receptor gamma $(P P A R G)$ represents a strong candidate gene predisposing to PCOS.

$P P A R G$ is crucial in the expression of key genes involved in adipose tissue differentiation, lipid and glucose metabolism, insulin sensitization, adipokine production and inflammation $(7,8)$. Several single-nucleotide polymorphisms (SNPs) of $P P A R G$, of which the PPARG Pro12Ala is the most extensively investigated, were found to reduce the transcriptional activity of PPARG, resulting in less active PPARG isoforms $(9,10)$. Previous studies demonstrated that the PPARG Pro12Ala variant allele is associated with higher insulin sensitivity, lower body mass index (BMI) (11), decreased risk of T2DM (12-14), higher high-density cholesterol (HDL) levels (11) and decreased incidence of CVD (15). Additionally, it was demonstrated that the PPARG Pro12Ala polymorphism may alter the plasma levels of leptin, adiponectin, resistin and ghrelin (16-19). 
The aim of the present study was to evaluate the frequency of PPARG Pro12Ala polymorphism in a Croatian population of PCOS patients and healthy controls. Additionally, we investigated whether the PCOS carriers of the PPARG X/Ala alleles differed in family and personal history, as well as in metabolic, biochemical and clinical parameters compared to the wild-type PPARG Pro/Pro carriers.

\section{Materials and methods}

Study subjects. This cohort was in part previously published (20). The patients were recruited from the gynecological endocrinology clinic of the University of Zagreb Medical Center, Zagreb, Croatia. A total of 151 PCOS patients were enrolled, with a mean age of $26.9 \pm 5.9$ years. The diagnosis of PCOS was confirmed according to the Rotterdam consensus criteria, based on the presence of two out of three traits, including oligo- and/or anovulation, clinical and/or biochemical signs of hyperandrogenism and the presence of polycystic ovaries on ultrasound scan (1). Other endocrinopathies and associated disorders were excluded by measuring basal serum follicle-stimulating hormone (FSH), luteinizing hormone ( $\mathrm{LH})$, prolactin, thyroid stimulating hormone and 17-hydroxyprogesterone levels. The menstrual irregularities were described as the presence of ameno- or oligomenorrhea. Hyperandrogenism was evaluated by the presence of hirsutism and/or acne and/or by elevated androgen levels [serum total testosterone $(\mathrm{TT})>2.5 \mathrm{nmol} / 1$ or free testosterone (FT) $>30 \mathrm{pmol} / \mathrm{l}$. Hirsutism was defined as a Ferriman-Gallwey score (FG) of $>8$ (21). All the patients enrolled in the study were evaluated during the early follicular phase of the menstrual cycle (days 3-5) or randomly in amenorrhoeic patients.

The information on T2DM and PCOS family history, low birth weight (LBW), childhood obesity, age of pubarche and menarche and presence of gestational diabetes (GDM) was obtained from the patients during a medical interview. The BMI was calculated as weight/height ${ }^{2}\left(\mathrm{~kg} / \mathrm{m}^{2}\right)$. The waist-to-hip ratio was calculated by dividing the waist circumference by the hip circumference. For the diagnosis of PCO according to the Rotterdam criteria, a transvaginal ultrasound scan was performed (1). The patients withheld any hormonal medications for $\geq 6$ months prior to enrollment in this study. Blood samples were drawn for measurements of $\mathrm{LH}, \mathrm{FSH}$, TT, sex hormone-binding globulin (SHBG), dehydroepiandrosterone sulfate (DHEA-S), androstenedione (A), fasting serum glucose, fasting insulin, total cholesterol, HDL, triglyceride, C-reactive protein (CRP), leptin, adiponectin, resistin and ghrelin levels. FT was calculated from TT and SHBG, as previously described (22), using a web-based calculator (http://www.issam.ch/freetesto.htm). Insulin resistance was quantified using the homeostatic model assessment of insulin resistance (HOMA-IR) [fasting insulin (mU/1) x fasting glucose (mmol/l)] /22.5]. The low-density cholesterol (LDL) concentration was calculated mathematically from total cholesterol, triglyceride and HDL cholesterol concentrations, using Friedewald's formula (23): LDL cholesterol $(\mathrm{mmol} / \mathrm{l})=$ total cholesterol (mmol/l) - HDL cholesterol (mmol/l) - triglycerides $(\mathrm{mmol} / \mathrm{l}) / 2.2$.

The control group included 179 healthy volunteers prior to entering an in vitro fertilisation program for male factor infertility, with a mean age of $29.2 \pm 4.7$ years. The healthy volunteers were free of menstrual cycle irregularities, clinical or biochemical hyperandrogenism, PCO on ultrasound scan, history of endocrinological or autoimmune disorders and surgery to the pelvic region. All the subjects enrolled in this study were of Croatian origin.

The study protocol (no. 04-1116-2006) was approved by the Ethics Committee of the University of Zagreb Medical School. Informed written consent was obtained from all the subjects enrolled in this study.

Hormone assays. The serum LH, FSH, SHBG, TT, DHEA-S and A levels were measured as previously described (20). The plasma glucose levels were determined by the UV-photometric hexokinase method and the serum insulin levels by the Immulite chemiluminescent immunometric assay (Siemens Healthcare Diagnostics, Inc., Deerfield, IL, USA). Total cholesterol and triglycerides were measured by standard routine enzymatic photometric assay. HDL cholesterol was determined following precipitation of very low-density lipoprotein and LDL with dextran sulphate and $\mathrm{MgCl}_{2}$. The CRP levels were determined by a particle-enhanced turbidimetric immunoassay technique, with the sensitivity of the test being $0.1 \mathrm{mg} / \mathrm{l}$. The serum leptin levels were measured by radioimmunoassay (RIA; DRG International, Springfield, NJ, USA). The analytical sensitivity of the method was $0.1 \mathrm{ng} / \mathrm{ml}$ (range, 0.1-100 $\mathrm{ng} / \mathrm{ml}$ ). The coefficient of variation from day to day was $\leq 5.8$ and $4.1 \%$ of the series. There was no cross-reaction with resistin, adiponectin and ghrelin. The serum adiponectin levels were measured by ELISA (Bio Vendor, Brno, Czech Republic). The analytical sensitivity of the method was 0.026 (range, $0.026-100 \mathrm{mg} / \mathrm{ml}$ ) and the coefficients of variation within the series were $\leq 5.9 \%$. The serum resistin levels were measured by enzyme immunoassay (DRG International). The analytical sensitivity of the method was $0.033 \mathrm{ng} / \mathrm{ml}$ (range, 0.033-50 ng/ml) and the coefficient of variation from day to day was $\leq 6.9$ and $3.4 \%$ of the series. The serum ghrelin levels were measured by RIA (DRG International). The analytical sensitivity of the method was 100 (range, 100-5,000 pg/ml) and the coefficient of variation from day to day was $\leq 7.7$ and $1.9 \%$ of the series.

Genotype analysis. Genotype analyses were conducted at the Department of Obstetrics and Gynaecology, Division of Medical Genetics, University of Ljubljana Medical Centre, Slovenia. Genomic DNA was isolated from whole blood using the FlexiGene DNA kit (Qiagen, Hilden, Germany) following the manufacturer's protocol. Genotyping for the PPARG Pro12Ala polymorphism (rs1801282) was performed with Custom TaqMan SNP Genotyping assay (Applied Biosystems, Foster City, CA, USA) on the ABI Prism 7900HT Sequence Detection system, using SDS 2.4 software (Applied Biosystems).

Statistical analysis. Data are expressed as percentages for categorical variables and as means \pm standard deviation for continuous variables. The association between PPARG Pro12Ala polymorphism and PCOS was assesed using logistic regression analysis, adjusted for age and BMI. We used the Mann-Whitney U test to compare continuous vari- 
Table I. Distribution of PPARG Pro12Ala alleles and genotypes in PCOS patients and controls.

\begin{tabular}{|c|c|c|c|c|c|c|c|}
\hline \multirow[b]{2}{*}{ Groups } & \multicolumn{2}{|c|}{ Alelle $(\%)$} & \multirow[b]{2}{*}{ P-value ${ }^{a}$} & \multicolumn{3}{|c|}{ Genotype (\%) } & \multirow[b]{2}{*}{ P-value } \\
\hline & Pro & Ala & & Pro/Pro & Pro/Ala & Ala/Ala & \\
\hline $\operatorname{PCOS}(n=151)$ & 84.4 & 15.6 & 0.787 & 70.2 & 28.5 & 1.3 & 0.880 \\
\hline Controls $(n=179)$ & 85.2 & 14.8 & & 72.1 & 26.3 & 1.7 & \\
\hline
\end{tabular}

${ }^{a} \chi^{2}$ test. PPARG, peroxisome proliferator-activated receptor gamma gene; PCOS, polycystic ovary syndrome.

ables between genotypes and the Chi-square test to compare categorical variables between genotypes.

The point-biserial correlation was used to assess the association between PPARG Pro12Ala polymorphism and insulin sensitivity. To further investigate the effect of the PPARG Pro12Ala polymorphism on insulin sensitivity measured as HOMA-IR, a multiple linear regression model was constructed with HOMA-IR as a dependent variable and PPARG Pro12Ala genotype (coded as Pro/Pro=1 and Pro/Ala+Ala/Ala=2), BMI, SHBG and TT as independent variables.

All the statistical analyses were performed using the SPSS software for Windows, version 15.0 (SPSS, Inc., Chicago, IL, USA). $\mathrm{P}<0.05$ was considered to indicate a statistically significant difference.

\section{Results}

Association of PPARG Prol2Ala polymorphism with PCOS. A total of 151 women with PCOS and 179 healthy volunteers were recruited in this study. The allele and genotype distributions of the PPARG Pro12Ala polymorphism for the PCOS and the control groups were consistent with the Hardy-Weinberg equilibrium (Table I). The distribution of the PPARG Pro12Ala alleles and genotypes was not significantly different between cases and controls, as presented in Table I $(\mathrm{P}=0.787$ and 0.880 , respectively).

Due to the low frequency of the homozygous Ala/Ala genotype, we divided genotypes into two categories: Pro/Pro and X/Ala (Pro/Ala+Ala/Ala). A logistic regression analysis was performed to assess the association of the PCOS status (coded as PCOS=0, controls=1) as the dependent variable and the PPARG Pro12Ala genotype (coded as Pro/Pro=1 and $\mathrm{X} / \mathrm{Ala}=2), \mathrm{BMI}$ and age as co-variables. The analysis demonstrated that the presence of the Ala allele is not associated with the PCOS status $(\mathrm{B}=0.136$ and $\mathrm{P}=0.594)$.

Association of PPARG Prol2Ala polymorphism with family and personal history, clinical, hormonal and metabolic parameters in $P C O S$. The anthropometric parameters, clinical characteristics, family and personal history in PCOS patients carrying the Pro/Pro or X/Ala genotypes are presented in Table II. We observed no significant differences between the genotype groups according to the traits regarding PCOS and the metabolic syndrome (Table II). The only significant difference between the PPARG Pro12Ala genotypes was in $\mathrm{BMI}$, which was higher in the Pro/Pro group $(\mathrm{P}=0.037)$. The hormonal and metabolic parameters according to the PPARG Pro12Ala genotypes are shown in Table III. No significant differences were observed in hormonal parameters, serum lipid levels, serum CRP levels or serum levels of leptin, adiponectin, resistin or ghrelin (Table III). We observed significantly higher serum fasting insulin levels and HOMA-IR in the PCOS patients who were carriers of the Pro/Pro allele compared to the X/Ala carriers $(\mathrm{P}<0.001)$. The point-biserial correlation analysis revealed a significant negative correlation between X/Ala and HOMA-IR $(\mathrm{R}=-0.236, \mathrm{P}=0.004)$. The equal analysis was conducted separately for the lean compared to the obese PCOS group. A significant negative correlation was found only in the lean PCOS group $(\mathrm{R}=-0.248, \mathrm{P}=0.007$ and $\mathrm{R}=-0.102, \mathrm{P}=0.559$, respectively). The presence of the polymorphic Ala allele was found to be a significant predictor of HOMA-IR variability, either unadjusted or following adjustment for BMI, SHBG and TT (Table IV). This multiple linear regression model explained $5.5 \%$ (adjusted $\mathrm{R}^{2}$ ) of the HOMA-IR variability (Table IV).

\section{Discussion}

In the present study, we evaluated the difference in frequency of the PPARG Pro12Ala polymorphism between PCOS patients and the healthy control group. To the best of our knowledge, this is the first study to investigate the association of the PPARG Pro12Ala polymorphism and PCOS in a Croatian population. We observed no significant difference in the distribution of the PPARG Pro12Ala allele between the investigated groups (Table I), indicating that the PPARG Pro12Ala is not a major determinant of PCOS in the Croatian population.

Although certain studies demonstrated a lower frequency of PPARG Pro12Ala polymorphism among PCOS patients (24-26), the majority of case-control studies (27-34) reported no significant differences. However, the merged results of several case-control studies revealed that the carriers of the polymorphic Ala allele have a lower risk of developing PCOS $(32,35,36)$. The difference between the findings of case studies and meta-analyses indicate that a very large sample size may be required to uncover the effect of genes that do not have a major genetic impact on PCOS, but rather represent genetically modifying factors.

We observed a high frequency of PPARG Pro12Ala polymorphism in the two investigated groups (30\% in PCOS patients and $28 \%$ in the controls, Table I). The frequency of PPARG Pro12Ala polymorphism among European PCOS patients ranges from 4.2 in the Italian population (31) to $22.9 \%$ in the Finnish population (25). Therefore, the prevalence of the PPARG Pro12Ala polymorphism in Croatian PCOS patients was found to be higher compared to that in the majority of 
Table II. Anthropometric parameters, clinical characteristics, family and personal history in PPARG Pro/Pro and PPARG X/Ala genotypes in PCOS patiens.

\begin{tabular}{|c|c|c|c|}
\hline Variables & Pro/Pro (n=106) & $\mathrm{X} / \mathrm{Ala}(\mathrm{n}=45)$ & P-value \\
\hline Age (years) & $26.9 \pm 5.9$ & $26.3 \pm 5.7$ & 0.529 \\
\hline $\mathrm{BMI}\left(\mathrm{kg} / \mathrm{m}^{2}\right)$ & $23.7 \pm 4.2$ & $22.6 \pm 4.1$ & $\mathbf{0 . 0 3 7}$ \\
\hline BMI>25 (kg/m²) $(\%)$ & 26.4 & 15.6 & 0.217 \\
\hline WHR & $0.79 \pm 0.08^{\mathrm{b}}$ & $0.78 \pm 0.07^{\mathrm{c}}$ & 0.700 \\
\hline Age at pubarche (years) & $11.2 \pm 1.6^{\mathrm{b}}$ & $10.9 \pm 1.5^{\mathrm{c}}$ & 0.647 \\
\hline Age at menarche (years) & $12.8 \pm 1.7^{\mathrm{b}}$ & $12.3 \pm 1.4^{\mathrm{c}}$ & 0.180 \\
\hline Family history of T2DM (\%) & $7.1^{\mathrm{b}}$ & $7.1^{\mathrm{c}}$ & 0.962 \\
\hline Family history of PCOS (\%) & $2.9^{\mathrm{b}}$ & $3.6^{\mathrm{c}}$ & 0.967 \\
\hline History of GDM (\%) & $1.4^{\mathrm{b}}$ & $10.7^{\mathrm{c}}$ & 0.106 \\
\hline Low birth weight (\%) & $5.7^{\mathrm{b}}$ & $3.5^{\mathrm{c}}$ & 0.885 \\
\hline Childhood obesity (\%) & $4.3^{\mathrm{b}}$ & $3.6^{\mathrm{c}}$ & 0.966 \\
\hline \multicolumn{4}{|l|}{ Hirsutism (\%) } \\
\hline FG 8-9 (\%) & $26.7 \mathrm{~b}$ & $22.6^{\mathrm{c}}$ & \\
\hline FG 10-14 (\%) & $36.7^{\mathrm{b}}$ & $35.5^{\mathrm{c}}$ & 0.928 \\
\hline $\mathrm{FG}>15(\%)$ & $16.7 b$ & $16.1^{\mathrm{c}}$ & \\
\hline FG-total score & $11.8 \pm 3.6^{\mathrm{b}}$ & $12.0 \pm 3.6^{\mathrm{c}}$ & 0.877 \\
\hline \multicolumn{4}{|l|}{ Acne $(\%)$} \\
\hline Mild (\%) & $26.7^{\mathrm{b}}$ & $29.0^{\mathrm{c}}$ & \\
\hline Moderate (\%) & $16.7^{\mathrm{b}}$ & $25.8^{\mathrm{c}}$ & 0.381 \\
\hline Severe $(\%)$ & $6.7^{\mathrm{b}}$ & $0.0^{\mathrm{c}}$ & \\
\hline \multicolumn{4}{|l|}{ Menstrual cycle } \\
\hline Normal (\%) & 11.7 & 6.5 & 0.713 \\
\hline Oligomenorrhea (\%) & 66.7 & 77.7 & \\
\hline Amenorrhea (\%) & 23.3 & 25.8 & \\
\hline
\end{tabular}

${ }^{a}$ The Mann-Whitney U test was used for continuous and the Chi-square test for categorical variables; ${ }^{\mathrm{b}} \mathrm{n}=60$; ${ }^{\mathrm{c}} \mathrm{n}=31$. $P P A R G$, peroxisome proliferator-activated receptor gamma gene; PCOS, polycystic ovary syndrome; BMI, body mass index; WHR, waist/hip ratio; T2DM, type 2 diabetes mellitus; GDM, gestational diabetes mellitus; FG, Ferriman-Gallwey score. Bold print denotes statistically significant differences.

the European population (25-29,31-34). To the best of our knowledge, the highest prevalence of the PPARG Pro12Ala polymorphism was reported in the Polish $(23,15 \%)(37)$ and the Slovenian populations $(27,5 \%)$ (38). These results may suggest that Slavic populations exhibit a higher occurrence of this polymorphic allele compared to the rest of the European PCOS population.

Furthermore, we assessed the effect of the PPARG Pro12Ala polymorphism on different metabolic, biochemical and clinical parameters, as well as on family and personal history in PCOS patients.

We observed significantly lower fasting insulin levels in the X/Ala compared to the Pro/Pro allele carriers $(\mathrm{P}<0.001)$, whereas the fasting glucose levels were comparable in the two groups $(\mathrm{P}=0.416)$. Consequently, HOMA-IR was also significantly lower $(\mathrm{P}<0.001)$. The adjusted linear regression model explained $5.5 \%$ of the variability in insulin sensitivity (expressed as HOMA-IR), where the presence of the polymorphic allele Ala was found to be the only significant predictor of HOMA-IR $(\mathrm{P}=0.008)$ (Table IV). We hypothesized that the presence of the polymorphic allele Ala represents a protective variant that alleviates hyperinsulinemia in Croatian women with PCOS who carry this allele. There is conflicting data in the literature regarding the association between PPARG Pro12Ala polymorphism and fasting insulin levels in PCOS patients. Certain studies $(27,32,38)$, but not others $(24,30,31,34,39)$ reported a significant association between the PPARG Pro12Ala polymorphism and fasting insulin levels. Moreover, a meta-analysis conducted by San-Millan and Escobar-Morreale (32), reported lower insulin levels in X/Ala carriers considering PCOS patients and controls as an entire group, although this effect was not observed in PCOS patients alone. Similar results were observed when analyzing the effect of the PPARG Pro12Ala on HOMA-IR. Two additional meta-analyses $(35,36)$ concluded that the PPARG Pro12Ala variant may result in lower insulin levels, but exerts no effect on HOMA-IR in PCOS patients. These conflicting results may be explained by the differences in the genetic background of the population investigated and by gene-gene and gene-environmental interactions (i.e., diet, exercise and age) (40).

The studies that evaluated normal and diabetic populations revealed that the $\mathrm{X} / \mathrm{Ala}$ is associated with reduced 
Table III. Hormonal and metabolic parameters in PPARG Pro/Pro and PPARG X/Ala genotypes in PCOS patients.

\begin{tabular}{|c|c|c|c|}
\hline Parameters & Pro/Pro $(n=106)$ & X/Ala $(n=45)$ & P-value ${ }^{a}$ \\
\hline FSH (IU/l) & $3.9 \pm 2.1$ & $3.8 \pm 1.8$ & 0.759 \\
\hline LH (IU/l) & $8.6 \pm 5.5$ & $9.6 \pm 7.0$ & 0.512 \\
\hline TT (nmol/l) & $2.3 \pm 0.9$ & $2.3 \pm 1.1$ & 0.588 \\
\hline FT (pmol/l) & $43.7 \pm 23.9$ & $40.2 \pm 21.8$ & 0.430 \\
\hline $\mathrm{A}(\mathrm{nmol} / \mathrm{l})$ & $11.9 \pm 5.7$ & $10.8 \pm 3.8$ & 0.436 \\
\hline DHEA-S $(\mu \mathrm{mol} / \mathrm{l})$ & $7.2 \pm 3.0$ & $6.1 \pm 2.6$ & 0.079 \\
\hline SHBG (nmol/1) & $34.3 \pm 22.1$ & $39.5 \pm 19.6$ & 0.105 \\
\hline Fasting insulin (pmol/l) & $11.6 \pm 7.7$ & $7.8 \pm 5.1$ & $<0.001$ \\
\hline Fasting glucose (mmol/l) & $4.3 \pm 0.6$ & $4.2 \pm 0.5$ & 0.416 \\
\hline HOMA-IR & $2.3 \pm 1.7$ & $1.5 \pm 1.0$ & $<0.001$ \\
\hline Total cholesterol (mmol/l) & $4.6 \pm 0.4^{\mathrm{b}}$ & $4.5 \pm 0.3^{c}$ & 0.522 \\
\hline HDL cholesterol (mmol/l) & $1.3 \pm 0.1^{\mathrm{b}}$ & $1.4 \pm 0.1^{\mathrm{c}}$ & 0.647 \\
\hline LDL cholesterol (mmol/l) & $2.7 \pm 0.4^{\mathrm{b}}$ & $2.6 \pm 0.4^{c}$ & 0.607 \\
\hline Triglycerides (mmol/l) & $1.4 \pm 0.4^{\mathrm{b}}$ & $1.3 \pm 0.3^{\mathrm{c}}$ & 0.434 \\
\hline CRP (mg/l) & $2.0 \pm 0.6^{b}$ & $2.2 \pm 0.6^{\mathrm{c}}$ & 0.103 \\
\hline Leptin (ng/ml) & $11.5 \pm 1.7^{\mathrm{b}}$ & $10.9 \pm 1.6^{\mathrm{c}}$ & 0.128 \\
\hline Adiponectin $(\mu \mathrm{g} / \mathrm{ml})$ & $9.1 \pm 1.7^{b}$ & $9.3 \pm 1.7^{\mathrm{c}}$ & 0.397 \\
\hline Resistin (ng/ml) & $12.4 \pm 2.3^{\mathrm{b}}$ & $11.7 \pm 2.5^{\mathrm{c}}$ & 0.247 \\
\hline Ghrelin (pg/ml) & $1,123.4 \pm 207.9^{b}$ & $1,234.9 \pm 237.1^{\mathrm{c}}$ & 0.057 \\
\hline
\end{tabular}

${ }^{a}$ Mann-Whitney U test; ${ }^{b} \mathrm{n}=45 ;{ }^{\mathrm{c}} \mathrm{n}=25$. PPARG, peroxisome proliferator-activated receptor gamma gene; PCOS, polycystic ovary syndrome; FSH, follicle-stimulating hormone; LH, luteinizing hormone; TT, total testosterone; FT, free testosterone; A, androstenedione; DHEA-S, dehydroepiandrosterone sulphate; SHBG, sex hormone-binding globulin; HOMA-IR, homeostatic model assessment of insulin resistance; HDL, high-density lipoprotein; LDL, low-density lipoprotein; CRP, C-reactive protein. Bold print denotes statistically significant differences.

Table IV. Multiple linear regression models with HOMA-IR as the dependent variable in PCOS patients.

\begin{tabular}{lll}
\hline Variables & B-coefficient $(95 \% \mathrm{CI})$ & P-value \\
\hline Unadjusted model $\left(\mathrm{R}^{2}=0.049\right)$ & & \\
PPARG Pro12Ala genotype & -0.81 (from 1.35 to -0.27$)$ & \\
Adjusted model $\left(\mathrm{R}^{2}=0.055\right)$ & & \\
PPARG Pro12Ala genotype & -0.74 (from -1.30 to -0.20$)$ & 0.008 \\
BMI & 0.03 (from -0.03 to 0.09$)$ & 0.281 \\
TT & -0.14 (from -0.40 to 0.11$)$ & 0.271 \\
SHBG & -0.01 (from -0.02 to 0.01$)$ & 0.266 \\
\hline
\end{tabular}

HOMA-IR, homeostatic model assessment of insulin resistance; PCOS, polycystic ovary syndrome; CI, confidence interval; PPARG, peroxisome proliferator-activated receptor gamma gene; BMI, body mass index; TT, total testosterone; SHBG, sex hormone-binding globulin.

weight and improved insulin sensitivity, predominantly in lean subjects $(11,12,41,42)$. Therefore, the X/Ala is viewed as being protective against T2DM in non-obese subjects $(11,12)$. However, in obese subjects, the effect of the X/Ala allele has not been fully elucidated $(41,43)$. It appears that obesity may mask the protective effect of the Ala allele (44). The majority of studies reported no apparent effect of the PPARG Pro12Ala genotype on BMI in PCOS patients $(24,27,29-31,34,39)$. We observed that the X/Ala genotype was associated with insulin sensitivity only in non-obese individuals, which is in accordance with the findings of studies conducted on Turkish (26) and Slovenian women (38) with PCOS. Therefore, the PPARG Pro12Ala genotype may be sensitive to environmental influences, specifically dietary ones (45). Free fatty acids that are generated in the process of nutrition and metabolism are natural ligands for PPARG (45). Luan et al (46) demonstrated that the consumption of a diet with a low polyunsaturated to saturated fat ratio was associated with lower insulin levels and BMI in X/Ala carriers compared to those in Pro/Pro carriers. Garaulet et al (47) also reported a protective role of the Ala allele against weight gain in individals practicing Mediterranean diet principles. The dietary habits of the patients were not analyzed 
in our study, although we hypothesized that a large proportion of patients follow the Mediterranean diet. Ranilovic et al (48) reported that Croats have the most similar dietary habits to the Italian population. The Mediterranean diet is rich in unsaturated omega-3 fatty acids, which may have affected our results. The high frequency of the PPARG Pro12Ala polymorphism and its protective effect on insulin sensitivity and BMI, together with the hypothesis of a Mediterranean diet, may explain why the Croatian population with PCOS was found to exhibit a low prevalence of insulin resistance and obesity, as demonstrated in our previous study (20).

A previous study reported elevated surrogate biomarkers for CVD in women with PCOS (6). CRP, a marker of low-grade chronic inflammation, represents a predictor of CVD (49) in PCOS patients. Yilmaz et al (26) and Tok et al (33) observed significantly lower CRP values in carriers of the Ala allele. We observed no effect of this SNP on CRP values in our study population, which is consistent with previous observations from the Slovenian population (38). Additionally, we observed no significant effect of the PPARG Pro12Ala polymorphism on plasma lipid levels in PCOS patients, which is in accordance with previous observations $(28,29,31)$.

Changes in adipokines may represent a link between insulin resistance, obesity and CVD. PPARG induces adipocyte differentiation and controls a number of adipocyte genes (40). Only a limited number of studies investigated the association between the PPARG Pro12Ala polymorphism and adipokines in PCOS patients $(28,31,50-53)$. No association between the serum leptin levels and the PPARG Pro12Ala polymorphism was observed in our study, which is consistent with the findings of two previous studies $(28,31)$. By contrast, Bidzinska-Speichert et al (37) reported a protective role of the X/Ala genotype on hyperleptinemia in women with PCOS. The PPARG Pro12Ala polymorphism exerted no effect on serum adiponectin levels in our cohort of PCOS patients, which was comparable to the findings of Orio et al (31) and Hahn et al (28). Similarly, we observed no association between the PPARG Pro12Ala polymorphism and the serum levels of resistin. Wang et al (54) reported that the carriers of the X/Ala allele exhibited lower plasma resistin levels in the Chinese population, whereas no difference was observed in a study from India (14). No effect of the PPARG Pro12Ala polymorphism on serum ghrelin concentrations was detected in the present study, confirming previously published data (28).

Ovarian hyperandrogenism is considered the fundamental abnormality of PCOS. We observed no association between the PPARG Pro12Ala polymorphism and serum androgen levels, which is in accordance with the observations of the majority of previously published studies $(24,27-29,33,34)$. Only two previous studies reported reduced serum androgen levels $(24,26)$ and hirsutism (24) in PCOS patients carrying the PPARG X/Ala genotype.

PPARG isoforms are located in the pilosebaceous unit (55), affect sebaceous cell growth and differentiation (56) and, therefore, may promote the cutaneous manifestations of hyperandrogenism in PCOS patients. The number of studies that evaluated the effect of the PPARG Pro12Ala polymorphism on clinical findings in PCOS patients is limited. Hahn et al (28), reported a lower hirsutism score in PCOS women carrying the Ala allele, implicating PPARGs as a possible novel local factor in hair growth. Conversely, we observed no effect of the PPARG Pro12Ala genotype on the prevalence and grade of acne and hirsutism. Therefore, the role of PPARGs in modulating androgen levels and its effect of terminal hair growth remains disputable.

LBW has been linked to cardiometabolic risk factors, including obesity, hypertension, dyslipidemia and T2DM in adulthood (57), particularly in those individuals with catch-up growth (58). Eriksson et al (59) reported that the Ala allele may protect against the insulin resistance that is associated with LBW. Furthermore, LBW has also been associated with early pubertal development in girls, followed by functional hyperandrogenism in adolescence (60). We observed no association between the presence of the PPARG Pro12Ala polymorphism and LBW, childhood obesity or premature pubarche. Furthermore, no association was observed between PPARG Pro12Ala polymorphism and family history for T2DM or PCOS.

In conclusion, the PPARG Pro12Ala polymorphism is not a major determinant of PCOS in the Croatian population; however, it exerts a positive effect on insulin sensitivity and BMI. As these associations were recorded exclusively in the lean group of PCOS patients, this polymorphism potentially exerts a protective effect against hyperinsulinemia and obesity.

\section{Acknowledgements}

We would like to thank Miss Iva Trgovcic for the revision of the English manuscript. This study was supported by a grant from the Ministry of Science, Education and Sports of the Republic of Croatia (no. 108-0000000-0388).

\section{References}

1. Rotterdam ESHRE/ASRM-Sponsored PCOS consensus workshop group: Revised 2003 consensus on diagnostic criteria and long-term health risks related to polycystic ovary syndrome (PCOS). Hum Reprod 19: 41-47, 2004.

2. Diamanti-Kandarakis E and Piperi C: Genetics of polycystic ovary syndrome: searching for the way out of the labyrinth. Hum Reprod Update 11: 631-643, 2005.

3. Alberti KG, Zimmet P and Shaw J: International Diabetes Federation: a consensus on Type 2 diabetes prevention. Diabet Med 24: 451-463, 2007.

4. Carmina E, Orio F, Palomba S, et al: Evidence for altered adipocyte function in polycystic ovary syndrome. Eur J Endocrinol 152: 389-394, 2005.

5. Ehrmann DA, Barnes RB, Rosenfield RL, Cavaghan MK and Imperial J: Prevalence of impaired glucose tolerance and diabetes in women with polycystic ovary syndrome. Diabetes Care 22: 141-146, 1999.

6. Schmidt J, Landin-Wilhelmsen K, Brannstrom M and Dahlgren E: Cardiovascular disease and risk factors in PCOS women of postmenopausal age: a 21-year controlled follow-up study. J Clin Endocrinol Metab 96: 3794-3803, 2011.

7. Kadowaki T, Hara K, Kubota N, et al: The role of PPARgamma in high-fat diet-induced obesity and insulin resistance. J Diabetes Complications 16: 41-45, 2002 .

8. Zhang J, Fu M, Cui T, et al: Selective disruption of PPARgamma 2 impairs the development of adipose tissue and insulin sensitivity. Proc Natl Acad Sci USA 101: 10703-10708, 2004.

9. Elbrecht A, Chen Y, Cullinan CA, et al: Molecular cloning, expression and characterization of human peroxisome proliferator activated receptors gamma 1 and gamma 2. Biochem Biophys Res Commun 224: 431-437, 1996.

10. Rangwala SM and Lazar MA: Peroxisome proliferator-activated receptor gamma in diabetes and metabolism. Trends Pharmacol Sci 25: 331-336, 2004. 
11. Deeb SS, Fajas L, Nemoto M, et al: A Pro12Ala substitution in PPARgamma2 associated with decreased receptor activity, lower body mass index and improved insulin sensitivity. Nat Genet 20 : 284-287, 1998

12. Altshuler D, Hirschhorn JN, Klannemark M, et al: The common PPARgamma Pro12Ala polymorphism is associated with decreased risk of type 2 diabetes. Nat Genet 26: 76-80, 2000.

13. Hara K, Okada T, Tobe K, et al: The Pro12Ala polymorphism in PPAR gamma2 may confer resistance to type 2 diabetes. Biochem Biophys Res Commun 271: 212-216, 2000.

14. Jacob S, Stumvoll M, Becker R, et al: The PPARgamma2 polymorphism pro12Ala is associated with better insulin sensitivity in the offspring of type 2 diabetic patients. Horm Metab Res 32: 413-416, 2000.

15. Doney AS, Fischer B, Leese G, Morris AD and Palmer CN Cardiovascular risk in type 2 diabetes is associated with variation at the PPARG locus: a Go-DARTS study. Arterioscler Thromb Vasc Biol 24: 2403-2407, 2004.

16. Demers A, Caron V, Rodrigue-Way A, Wahli W, Ong H and Tremblay A: A concerted kinase interplay identifies PPARgamma as a molecular target of ghrelin signaling in macrophages. PLoS One 4: e7728, 2009.

17. Haseeb A, Iliyas M, Chakrabarti S, et al: Single-nucleotide polymorphisms in peroxisome proliferator-activated receptor gamma and their association with plasma levels of resistin and the metabolic syndrome in a South Indian population. J Biosci 34: 405-414, 2009

18. Simon I, Vendrell J, Gutierrez C, et al: Pro12Ala substitution in the peroxisome proliferator-activated receptor-gamma is associated with increased leptin levels in women with type-2 diabetes mellitus. Horm Res 58: 143-149, 2002.

19. Yamamoto Y, Hirose H, Miyashita K, et al: PPAR(gamma)2 gene Pro12Ala polymorphism may influence serum level of an adipocyte-derived protein, adiponectin, in the Japanese population. Metabolism 51: 1407-1409, 2002.

20. Baldani DP, Skrgatic L, Goldstajn MS, et al: Clinical and biochemical characteristics of polycystic ovary syndrome in Croatian population. Coll Antropol 36: 1413-1418, 2012.

21. Ferriman D and Gallwey JD: Clinical assessment of body hair growth in women. J Clin Endocrinol Metab 21: 1440-1447, 1961

22. Vermeulen A, Verdonck L and Kaufman JM: A critical evaluation of simple methods for the estimation of free testosterone in serum. J Clin Endocrinol Metab 84: 3666-3672, 1999.

23. Friedewald WT, Levy RI and Fredrickson DS: Estimation of the concentration of low-density lipoprotein cholesterol in plasma, without use of the preparative ultracentrifuge. Clin Chem 18: 499-502, 1972.

24. Hara M, Alcoser SY, Qaadir A, Beiswenger KK, Cox NJ and Ehrmann DA: Insulin resistance is attenuated in women with polycystic ovary syndrome with the Pro(12)Ala polymorphism in the PPARgamma gene. J Clin Endocrinol Metab 87: 772-775, 2002.

25. Korhonen S, Heinonen S, Hiltunen M, et al: Polymorphism in the peroxisome proliferator-activated receptor-gamma gene in women with polycystic ovary syndrome. Hum Reprod 18 540-543, 2003

26. Yilmaz M, Ergun MA, Karakoc A, Yurtcu E, Cakir N and Arslan M: Pro12Ala polymorphism of the peroxisome proliferator-activated receptor-gamma gene in women with polycystic ovary syndrome. Gynecol Endocrinol 22: 336-342, 2006.

27. Christopoulos P, Mastorakos G, Gazouli M, et al: Peroxisome proliferator-activated receptor-gamma and -delta polymorphisms in women with polycystic ovary syndrome. Ann NY Acad Sci 1205: 185-191, 2010.

28. Hahn S, Fingerhut A, Khomtsiv U, et al: The peroxisome proliferator activated receptor gamma Pro12Ala polymorphism is associated with a lower hirsutism score and increased insulin sensitivity in women with polycystic ovary syndrome. Clin Endocrinol (Oxf) 62: 573-579, 2005.

29. Koika V, Marioli DJ, Saltamavros AD, et al: Association of the Pro12Ala polymorphism in peroxisome proliferator-activated receptor gamma2 with decreased basic metabolic rate in women with polycystic ovary syndrome. Eur J Endocrinol 161: 317-322, 2009.

30. Orio F Jr, Matarese G, Di Biase S, et al: Exon 6 and 2 peroxisome proliferator-activated receptor-gamma polymorphisms in polycystic ovary syndrome. J Clin Endocrinol Metab 88: 5887-5892, 2003.

31. Orio F Jr, Palomba S, Cascella T, et al: Lack of an association between peroxisome proliferator-activated receptor-gamma gene Pro12Ala polymorphism and adiponectin levels in the polycystic ovary syndrome. J Clin Endocrinol Metab 89: 5110-5115, 2004.
32. San-Millan JL and Escobar-Morreale HF: The role of genetic variation in peroxisome proliferator-activated receptors in the polycystic ovary syndrome (PCOS): an original case-control study followed by systematic review and meta-analysis of existing evidence. Clin Endocrinol (Oxf) 72: 383-392, 2010.

33. Tok EC, Aktas A, Ertunc D, Erdal EM and Dilek S: Evaluation of glucose metabolism and reproductive hormones in polycystic ovary syndrome on the basis of peroxisome proliferator-activated receptor (PPAR)-gamma2 Pro12Ala genotype. Hum Reprod 20: 1590-1595, 2005.

34. Xita N, Lazaros L, Georgiou I and Tsatsoulis A: The Pro12Ala polymorphism of the PPAR-gamma gene is not associated with the polycystic ovary syndrome. Hormones (Athens) 8: 267-272, 2009.

35. He J, Wang L, Liu J, Liu F and Li X: A meta-analysis on the association between PPAR-gamma Pro12Ala polymorphism and polycystic ovary syndrome. J Assist Reprod Genet 29: 669-677, 2012.

36. Zhang H, Bi Y, Hu C, Lu W and Zhu D: Association between the Prol2Ala polymorphism of PPAR-gamma gene and the polycystic ovary syndrome: a meta-analysis of case-control studies. Gene 503: 12-17, 2012.

37. Bidzinska-Speichert B, Demissie M, Tworowska U, et al: Leptin level and the PPARgamma2 Pro12Ala and Pro115Gln polymorphisms in women with functional hyperandrogenism. Preliminary report. Przegl Lek 62: 833-837, 2005 (In Polish).

38. Dragojevic J, Ostanek B, Mencej-Bedrac S, Komadina R, Prezelj J and Marc J: PPARG gene promoter polymorphism is associated with non-traumatic hip fracture risk in the elderly Slovenian population: a pilot study. Clin Biochem 44: 1085-1089, 2011.

39. Antoine HJ, Pall M, Trader BC, Chen YD, Azziz R and Goodarzi MO: Genetic variants in peroxisome proliferator-activated receptor gamma influence insulin resistance and testosterone levels in normal women, but not those with polycystic ovary syndrome. Fertil Steril 87: 862-869, 2007.

40. Lehrke $M$ and Lazar MA: The many faces of PPARgamma. Cell 123: 993-999, 2005.

41. Ek J, Urhammer SA, Sorensen TI, Andersen T, Auwerx J and Pedersen O: Homozygosity of the Pro12Ala variant of the peroxisome proliferation-activated receptor-gamma2 (PPAR-gamma2): divergent modulating effects on body mass index in obese and lean Caucasian men. Diabetologia 42: 892-895, 1999.

42. Kao WH, Coresh J, Shuldiner AR, et al; Atherosclerosis Risk in Communities Study: Pro12Ala of the peroxisome proliferator-activated receptor-gamma2 gene is associated with lower serum insulin levels in nonobese African Americans: the Atherosclerosis Risk in Communities Study. Diabetes 52: $1568-1572,2003$

43. Masud S, Ye S and SAS Group: Effect of the peroxisome proliferator activated receptor-gamma gene Pro12Ala variant on body mass index: a meta-analysis. J Med Genet 40: 773-780, 2003.

44. Jaziri R, Lobbens S, Aubert R, et al; DESIR Study Group: The PPARG Pro12Ala polymorphism is associated with a decreased risk of developing hyperglycemia over 6 years and combines with the effect of the APM1 G-11391A single nucleotide polymorphism: the Data From an Epidemiological Study on the Insulin Resistance Syndrome (DESIR) study. Diabetes 55: $1157-1162,2006$

45. Huguenin GV and Rosa G: The Ala allele in the PPAR-gamma2 gene is associated with reduced risk of type 2 diabetes mellitus in Caucasians and improved insulin sensitivity in overweight subjects. Br J Nutr 104: 488-497, 2010.

46. Luan J, Browne PO, Harding AH, et al: Evidence for gene-nutrient interaction at the PPARgamma locus. Diabetes 50: 686-689, 2001.

47. Garaulet M, Smith CE, Hernandez-Gonzalez T, Lee YC and Ordovas JM: PPARgamma Pro12Ala interacts with fat intake for obesity and weight loss in a behavioural treatment based on the Mediterranean diet. Mol Nutr Food Res 55: 1771-1779, 2011.

48. Ranilovic J, Markovina J, Znidar K and Colic Baric I: Attitudes to healthy eating among a representative sampling of Croatian adults: a comparison with Mediterranean countries. Int J Food Sci Nutr 60: 11-29, 2009.

49. Boulman N, Levy Y, Leiba R, et al: Increased C-reactive protein levels in the polycystic ovary syndrome: a marker of cardiovascular disease. J Clin Endocrinol Metab 89: 2160-2165, 2004.

50. Bidzinska-Speichert B, Lenarcik A, Tworowska-Bardzinska U, et al: Pro12Ala PPAR gamma2 gene polymorphism in women with polycystic ovary syndrome. Ginekol Pol 82: 426-429, 2011. 
51. Kondo H, Shimomura I, Matsukawa Y, et al: Association of adiponectin mutation with type 2 diabetes: a candidate gene for the insulin resistance syndrome. Diabetes 51: 2325-2328, 2002.

52. Kubota N, Terauchi Y, Yamauchi T, et al: Disruption of adiponectin causes insulin resistance and neointimal formation. J Biol Chem 277: 25863-25866, 2002.

53. Weyer C, Funahashi T, Tanaka S, et al: Hypoadiponectinemia in obesity and type 2 diabetes: close association with insulin resistance and hyperinsulinemia. J Clin Endocrinol Metab 86: 1930-1935, 2001

54. Wang C, Zhai F, Chi Y and Wang G: Association of Pro12Ala mutation in peroxisome proliferator-activated receptor gamma 2 with obesity and diabetes in Chinese population. J Hyg Res 33: 317-320, 2004 (In Chinese).

55. Botchkarev VA and Paus R: Molecular biology of hair morphogenesis: development and cycling. J Exp Zool B Mol Dev Evol 298: 164-180, 2003.

56. Smith KJ, Dipreta E and Skelton H: Peroxisomes in dermatology. Part II. J Cutan Med Surg 5: 315-322, 2001.
57. Sas T, Mulder P, Aanstoot HJ, et al: Carbohydrate metabolism during long-term growth hormone treatment in children with short stature born small for gestational age. Clin Endocrinol (Oxf) 54: 243-251, 2001

58. Leunissen RW, Oosterbeek P, Hol LK, Hellingman AA, Stijnen T and Hokken-Koelega AC: Fat mass accumulation during childhood determines insulin sensitivity in early adulthood. J Clin Endocrinol Metab 93: 445-451, 2008.

59. Eriksson JG, Lindi V, Uusitupa M, et al: The effects of the Prol2Ala polymorphism of the peroxisome proliferator-activated receptor-gamma2 gene on insulin sensitivity and insulin metabolism interact with size at birth. Diabetes 51: 2321-2324, 2002.

60. Ibanez L, Potau N, Francois I and de Zegher F: Precocious pubarche, hyperinsulinism, and ovarian hyperandrogenism in girls: relation to reduced fetal growth. J Clin Endocrinol Metab 83: 3558-3562, 1998. 